\title{
Similarity reduction of the modified Yajima-Oikawa equation
}

\author{
Tetsuya KIKUCHI ${ }^{1 *}$ Takeshi $\operatorname{IKEDA}^{2 \dagger}$ and Saburo KAKEI ${ }^{3 \ddagger}$
}

August 29, 2003

\author{
${ }^{1}$ Mathematical Institute, Tohoku University, \\ Sendai 980-8578, JAPAN \\ ${ }^{2}$ Department of Applied Mathematics, Okayama University of Science, \\ Okayama 700-0005, JAPAN \\ ${ }^{3}$ Department of Mathematics, Rikkyo University, \\ Tokyo 171-8501, JAPAN
}

\begin{abstract}
We study a similarity reduction of the modified Yajima-Oikawa hierarchy. The hierarchy is associated with a non-standard Heisenberg subalgebra in the affine Lie algebra of type $A_{2}^{(1)}$. The system of equations for self-similar solutions is presented as a Hamiltonian system of degree of freedom two, and admits a group of Bäcklund transformations isomorphic to the affine Weyl group of type $A_{2}^{(1)}$. We show that the system is equivalent to a two-parameter family of the fifth Painlevé equation.
\end{abstract}

\section{Introduction}

In applications of the theory of affine Lie algebras to integrable hierarchies, the Heisenberg subalgebras play important roles, since they correspond to the varieties of time-evolutions. Let $\hat{\mathfrak{g}}$ be the untwisted affine Lie algebra associated with a finite-dimensional simple Lie algebra $\mathfrak{g}$. Up to conjugacy, the Heisenberg subalgebras in $\hat{\mathfrak{g}}$ are in one-to-one correspondence with the conjugacy classes of the Weyl group of $\mathfrak{g}[3$. In particular, the conjugacy class containing the Coxeter element, to which the principal Heisenberg subalgebra of $\hat{\mathfrak{g}}$ is associated, leads to the Drinfel'd-Sokolov hierarchy [2], whereas the class of the identity element corresponds to the homogeneous Heisenberg subalgebra. Associated with arbitrary conjugacy class, M. F. de Groot, T. J. Hollowood, J. L. Miramontes [1] developed the theory of integrable systems called generalized Drinfel'd-Sokolov hierarchies.

\footnotetext{
${ }^{*}$ E-mail address : tkikuchi@math.tohoku.ac.jp,

${ }^{\dagger} E$-mail address : ike@xmath.ous.ac.jp

${ }^{\ddagger} E$-mail address : kakei@rkmath.rikkyo.ac.jp
} 
When $\mathfrak{g}$ is of type $A_{n-1}$, the conjugacy classes are parametrized by the partitions of $n$. In this paper we consider the modified Yajima-Oikawa hierarchy, which turns out to be a hierarchy related to the affine Lie algebra of type $A_{2}^{(1)}$ and its non-standard Heisenberg subalgebra associated with the partition $(2,1)$, while the principal (resp. homogeneous ) case corresponds to the partition (3) (resp. $(1,1,1)$ ).

Among the issues on integrable hierarchies, the study of similarity reduction is important. For example, M. Noumi and Y. Yamada introduced a higher order Painlevé system associated with the affine root system of type $A_{n-1}^{(1)}[7]$ and now the system is known to be equivalent to a similarity reduction of the system associated with the Coxeter class $(n)$ of $A_{n-1}$. The aim of this paper is to investigate a similarity reduction of the modified Yajima-Oikawa hierarchy. Starting with universal viewpoints, we derive a system of ordinary differential equations for unknown functions $f_{0}, f_{1}, f_{2}, u_{0}, u_{1}, u_{2}, g, q, r$ and complex paremeters $\alpha_{0}, \alpha_{1}, \alpha_{2}$ :

$$
\begin{array}{ll}
\alpha_{0}^{\prime}=\alpha_{1}^{\prime}=\alpha_{2}^{\prime}=0, & \\
f_{0}^{\prime}=f_{0}\left(u_{2}-u_{0}\right)-\alpha_{0}, & g^{\prime}=g\left(u_{0}-u_{2}\right)-q f_{1}+r f_{2}+\alpha_{0}+4, \\
f_{1}^{\prime}=f_{1}\left(u_{0}-u_{1}\right)-r \alpha_{1}, & 3 q^{\prime}=3 q\left(u_{1}-u_{0}\right)+q f_{0}-f_{2}, \\
f_{2}^{\prime}=f_{2}\left(u_{1}-u_{2}\right)-q \alpha_{2}, & 3 r^{\prime}=3 r\left(u_{2}-u_{1}\right)-r f_{0}+f_{1} .
\end{array}
$$

where $^{\prime}=d / d x$ denote the derivative with respect to the independent variable $x$. Under the algebraic relations

$$
\begin{gathered}
\alpha_{0}+\alpha_{1}+\alpha_{2}=-4, \quad g=f_{0}+3 q r, \quad u_{0}+u_{1}+u_{2}=0, \quad u_{1}=q r, \\
2 g u_{0}=q f_{1}-r f_{2}-g q r-\alpha_{0}-2,
\end{gathered}
$$

the system (1.1) turns out to be equivalent to the fifth Painlevé equation for $y=$ $-f_{0} /\left(3 u_{1}\right)$ :

$$
y^{\prime \prime}=\left(\frac{1}{2 y}+\frac{1}{y-1}\right)\left(y^{\prime}\right)^{2}-\frac{y^{\prime}}{x}+\frac{(y-1)^{2}}{x^{2}}\left(A y+\frac{B}{y}\right)+\frac{C}{x} y+D \frac{y(y+1)}{(y-1)},
$$

where the change of variable $x \rightarrow x^{2}$ is employed and the parameters are given by

$$
A=\frac{1}{2}\left(\frac{\alpha_{2}-\alpha_{1}}{12}\right)^{2}, \quad B=-\frac{1}{2}\left(\frac{\alpha_{0}}{4}\right)^{2}, \quad C=-\frac{\alpha_{2}-\alpha_{1}}{18}, \quad D=-\frac{1}{18} .
$$

On introducing the system (1.1), we shall describe the system in three ways:

1. Compatibility condition for a system of linear differential equations (Section 5),

2. A Hamiltonian system whose degree of freedom is two (Theorem 2),

3. Hirota bilinear equations for $\tau$-functions (Theorem 3 ).

The system (1.1) has a symmetry of the affine Weyl group of type $A_{2}^{(1)}$ as a group of Bäcklund transformations. First we give the symmetry as the compatibility of gauge transformations of linear differential equations and state it in the automorphism of the differential field

$$
K=\mathbf{C}\left(\alpha_{0}, \alpha_{1}, \alpha_{2}, f_{0}, f_{1}, f_{2}, g, q, r, u_{0}, u_{1}, u_{2}\right)
$$


with the derivation ' $: K \rightarrow K$ defined by (1.1) and algebraic relations (1.2) (Theorem 1). Then we extend the action of affine Weyl group on $K$ to the extended field $\widehat{F}$ of $K$ :

$$
\widehat{F}=\mathbf{C}\left(\alpha_{0}, \alpha_{1}, \alpha_{2}, x ; \tau_{0}, \tau_{1}, \tau_{2}, \sigma_{1}, \sigma_{2}, \tau_{0}^{\prime}, \tau_{1}^{\prime}, \tau_{2}^{\prime}, \sigma_{1}^{\prime}, \sigma_{2}^{\prime}\right)
$$

as a Bäcklund transformations, which is discussed in section 11] (Theorem 51).

The paper is organized as follows. In Sect 2, we review the notation related to the affine Lie algebra of type $A_{2}^{(1)}$. On the basis of the affine Lie algebra, we introduce the modified Yajima-Oikawa hierarchy in Sect 3. In Sect 4, we consider a condition of selfsimilarity on the solutions of the hierarchy. This condition yields a system of ordinary diferential equations, which is a main object in this paper. In Sect 5 the condition of self-similarity is also presented as a Lax-type equation. In Sect 6, we give a Weyl group symmetry of the system as a gauge transformation of the Lax equatoin (Theorem 11). In Sect [7 a Hamiltonian structure is introduced (Theorem 2). In Sect 8 we prove that our system is equivalent to a two-parameter family of the fifth Painlevé equation. In Sect 9 we introduce a set of $\tau$-functions and give a bilinear form of differential system (Theorem 3). Then in Sect 10 we lift the action of Weyl group to the $\tau$-functions (Theorem 4) and give a Jacobi-Trudi type formula (10.4) for the Weyl group orbit of the $\tau$-functions. In Sect 11, we prove that the Weyl group action on the $\tau$-functions commute with the derivation ${ }^{\prime}=d / d x$.

\section{Preliminaries on the affine Lie algebra of type $A_{2}^{(1)}$}

In this section, we collect necessary notions about the affine Lie algebra of type $A_{2}^{(1)}$. We mainly follow the notation used in [4], to which one should refer for further details.

Let $\mathfrak{g}=\mathfrak{s l}_{3}$. The affine Lie algebra $\hat{\mathfrak{g}}$ is realized as a central extension of the loop algebra $L \mathfrak{g}=\mathfrak{s l}_{3}\left(\mathbf{C}\left[z, z^{-1}\right]\right)$, together with the derivation $d=z \partial_{z}$

$$
\hat{\mathfrak{g}}=\mathfrak{s l}_{3}\left(\mathbf{C}\left[z, z^{-1}\right]\right) \oplus \mathbf{C} c \oplus \mathbf{C} d
$$

where $c$ denotes the canonical central element. Let us define the Chevalley generators $E_{i}, F_{i}, H_{i}(i=0,1,2)$ for the affine Lie algebra $\hat{\mathfrak{g}}$ by

$$
\begin{gathered}
E_{0}=z E_{3,1}, E_{1}=E_{1,2}, E_{2}=E_{2,3}, F_{0}=z^{-1} E_{1,3}, F_{1}=E_{2,1}, F_{2}=E_{3,2}, \\
H_{0}=c+E_{3,3}-E_{1,1}, \quad H_{1}=E_{1,1}-E_{2,2}, \quad H_{2}=E_{2,2}-E_{3,3},
\end{gathered}
$$

where $E_{i, j}$ is the matrix unit $E_{i, j}=\left(\delta_{i a} \delta_{j b}\right)_{a, b=1}^{3}$. The Cartan subalgebra of $\hat{\mathfrak{g}}$ is defined as $\hat{\mathfrak{h}}=\bigoplus_{i=0}^{2} \mathbf{C} H_{i} \oplus \mathbf{C} d$. We introduce the simple roots $\alpha_{j}$ and the fundamental weights $\Lambda_{j}$ as the following linear functionals on the Cartan subalgebra $\hat{\mathfrak{h}}$ :

$$
\left\langle H_{i}, \alpha_{j}\right\rangle=a_{i j}, \quad\left\langle H_{i}, \Lambda_{j}\right\rangle=\delta_{i j} \quad(i=0,1,2), \quad\left\langle d, \alpha_{j}\right\rangle=\delta_{0 j}, \quad\left\langle d, \Lambda_{j}\right\rangle=0
$$

for $j=0,1,2$, where $\left(a_{i j}\right)_{i=0}^{3}$ is the generalized Cartan matrix of type $A_{2}^{(1)}$ defined by

$$
\left[\begin{array}{ccc}
2 & -1 & -1 \\
-1 & 2 & -1 \\
-1 & -1 & 2
\end{array}\right]
$$


We define a non degenerate symmetric bilinear form $(. \mid$.$) on V=\hat{\mathfrak{h}}^{*}$ as follows:

$$
\left(\alpha_{i} \mid \alpha_{j}\right)=a_{i j}, \quad\left(\alpha_{i} \mid \Lambda_{0}\right)=\delta_{i 0}, \quad\left(\Lambda_{0} \mid \Lambda_{0}\right)=0 .
$$

We define simple reflections $s_{i}(i=0,1,2)$ by

$$
s_{i}(\lambda)=\lambda-\left\langle H_{i}, \lambda\right\rangle \alpha_{i}, \quad \lambda \in V
$$

They satisfy the fundamental relations

$$
s_{i}^{2}=1, \quad s_{i} s_{i+1} s_{i}=s_{i+1} s_{i} s_{i+1} \quad(i=0,1,2),
$$

where the indices are understood as elements of $\mathbf{Z} / 3 \mathbf{Z}$. Consider the group

$$
W=\left\langle s_{0}, s_{1}, s_{2}\right\rangle \subset \mathrm{GL}(V)
$$

generated by the simple reflections. The group $W$ is called the affine Weyl group of type $A_{2}^{(1)}$.

\section{Modified Yajima-Oikawa hierarchy}

In this section we introduce the modified Yajima-Oikawa hierarchy as generalized Drinfel'dSokolov reduction associated to the loop algebra $L \mathfrak{g}=\mathfrak{s l}_{3}\left(\mathbf{C}\left[z, z^{-1}\right]\right)$, following [1]. Let us introduce the following derivation on $L \mathfrak{g}$ :

$$
D=4 z \frac{\partial}{\partial z}-\operatorname{diag}(-1,0,1)
$$

Set

$$
L \mathfrak{g}_{j}=\{A \in L \mathfrak{g} \mid[D, A]=j A\} .
$$

Then we have a $\mathbf{Z}$-gradation $L \mathfrak{g}=\oplus_{j} L \mathfrak{g}_{j}$. Note that

$$
\operatorname{deg}\left(E_{0}\right)=-\operatorname{deg}\left(F_{0}\right)=2, \quad \operatorname{deg}\left(E_{j}\right)=-\operatorname{deg}\left(F_{j}\right)=1 \quad(j=1,2) .
$$

Consider the particular element

$$
\gamma=\left[\begin{array}{lll}
0 & 0 & 1 \\
0 & 0 & 0 \\
z & 0 & 0
\end{array}\right]
$$

and let $\mathfrak{s}$ be the centralizer of $\gamma$ in $L \mathfrak{g}$

$$
\mathfrak{s}=\operatorname{Ker}(\operatorname{ad} \gamma)=\{A \in L \mathfrak{g} \mid[\gamma, A]=0\}
$$

The subalgebra $\mathfrak{s}$ is a maximal commutative subalgebra in $\mathfrak{g}$, which has the following basis:

$$
\gamma_{4 j+2}=z^{j} \gamma, \quad \gamma_{4 j}=z^{j} \operatorname{diag}(1,-2,1) \quad(j \in \mathbf{Z})
$$


Then $\mathfrak{s}$ is a graded subalgebra of $L \mathfrak{g}$ with respect to the gradation. We have $\gamma_{2 j} \in L \mathfrak{g}_{2 j}$. The commutative subalgebra $\mathfrak{s}$ is the image of a Heisenberg subalgebra in $\hat{\mathfrak{g}}$ associated with the conjugacy class $(2,1)([3]$, see also [10] and [5] $)$. We put $\mathfrak{b}:=\oplus_{j \geq 0} L \mathfrak{g}_{j}$.

To introduce our hierarchy, we begin with the differential operator

$$
L:=\frac{\partial}{\partial x}-\gamma-Q
$$

where $Q$ is an $x$-dependent element of $\mathfrak{b}_{<2}$. We set $\mathfrak{s}^{\perp}:=\operatorname{Im}(\operatorname{ad} \gamma)$. It is clear that $\mathfrak{s}^{\perp}=$ $\oplus_{j} \mathfrak{s}_{j}^{\perp}$, where $\mathfrak{s}_{j}^{\perp}:=\mathfrak{s}^{\perp} \cap L \mathfrak{g}_{j}$. There is a unique formal series $U=\sum_{j=1}^{\infty} U_{-j}\left(U_{-j} \in \mathfrak{s}_{-j}^{\perp}\right)$ such that the operator $L_{0}:=e^{\operatorname{ad} U}(L)$ has the form

$$
L_{0}=\frac{\partial}{\partial x}-\gamma-\sum_{j=0}^{\infty} h_{-2 j}, \quad h_{-2 j} \in \mathfrak{s}_{-2 j} .
$$

Moreover $U_{-j}$ and $h_{-2 j}$ are polynomials in the components of $Q$ and their $x$ derivatives. For any $j>0$ we set

$$
B_{2 j}=\left(e^{-\operatorname{ad} U} \gamma_{2 j}\right)_{\geq 0} .
$$

The modified Yajima-Oikawa hierarchy is defined by the Lax equations

$$
\frac{\partial L}{\partial t_{2 j}}=\left[B_{2 j}, L\right] \quad(j=1,2, \ldots) .
$$

We describe the above construction concretely. First we set

$$
Q=\left[\begin{array}{ccc}
u_{0} & r & 0 \\
0 & u_{1} & q \\
0 & 0 & u_{2}
\end{array}\right], \quad u_{0}+u_{1}+u_{2}=0
$$

and solve for the first few terms of $U_{j}$ and $h_{j}$ :

$$
\begin{aligned}
U_{-1}= & -q E_{2,1}+r E_{3,2}, \\
U_{-2}= & \frac{u_{2}-u_{0}}{4}\left(z^{-1} E_{1,3}-E_{3,1}\right), \\
U_{-3}= & {\left[\left(\frac{3 u_{0}}{8}+\frac{3 u_{1}}{2}-\frac{3 u_{2}}{8}-q r\right) r+r^{\prime}\right] E_{1,2} } \\
& \quad+\left[\left(\frac{7 u_{0}}{8}-u_{1}+\frac{u_{2}}{8}+q r\right) q+q^{\prime}\right] E_{2,3}, \\
U_{-4}= & {\left[\frac{u_{0}^{\prime}-u_{2}^{\prime}}{8}+\frac{q^{\prime} r+3 q r^{\prime}}{8}+\left(\frac{u_{0}}{16}-\frac{5 u_{1}}{16}+\frac{u_{2}}{16}+\frac{5}{16} q r\right) q r\right]\left(E_{1,1}-E_{3,3}\right), } \\
h_{0}= & \frac{q r-u_{1}}{2} \gamma_{0}, \\
h_{-2}= & {\left[\frac{u_{0}^{2}+u_{2}^{2}}{8}-\frac{u_{0} u_{2}}{4}-\frac{q^{\prime} r+3 q r^{\prime}}{4}-\left(\frac{u_{0}}{8}-\frac{5 u_{1}}{8}+\frac{u_{2}}{8}+\frac{3}{8} q r\right) q r\right] \gamma_{-2} . }
\end{aligned}
$$


Here ' means $\partial / \partial x$. In fact, $h_{0}$ is a constant along all the flows and we can put $h_{0}=0$ (see [1]). So we fix

$$
u_{1}=q r
$$

from now on. By using $U_{j}$ 's and condition (3.2) we have

$$
\begin{gathered}
B_{2}=\gamma_{2}+\left[\begin{array}{ccc}
u_{0} & r & 0 \\
0 & u_{1} & q \\
0 & 0 & u_{2}
\end{array}\right], \\
B_{4}=\gamma_{4}+3\left[\begin{array}{ccc}
-q r^{\prime}+q r u_{2} & r^{\prime}-r u_{2} & 0 \\
q z & q r^{\prime}-q^{\prime} r+q r u_{1} & -q^{\prime}-q u_{0} \\
-q r z & r z & q^{\prime} r+q r u_{0}
\end{array}\right]
\end{gathered}
$$

The modified Yajima-Oikawa equation is obtained by the following zero-curvature condition:

$$
\frac{\partial B_{2}}{\partial t_{4}}=\frac{\partial B_{4}}{\partial t_{2}}-\left[B_{2}, B_{4}\right]
$$

In fact this yields the following system of differential equations:

$$
\begin{gathered}
q_{t}+3\left(q^{\prime \prime}+q\left(-q r^{\prime}+u_{0}^{\prime}+q r u_{2}+u_{2}^{2}\right)\right)=0, \\
r_{t}-3\left(r^{\prime \prime}-r\left(-q^{\prime} r+u_{2}^{\prime}-q r u_{0}+u_{0}^{2}\right)\right)=0, \\
\left(u_{0}\right)_{t}=3\left(-q r^{\prime}+q r u_{2}\right)^{\prime}, \quad\left(u_{1}\right)_{t}=3\left(q r^{\prime}-q^{\prime} r+q r u_{1}\right)^{\prime}, \quad\left(u_{2}\right)_{t}=3\left(q^{\prime} r+q r u_{0}\right)^{\prime} .
\end{gathered}
$$

Here we identify $x$ and $t_{2}$, and put $t=t_{4}$.

Remark: This system of equations is related to the Yajima-Oikawa equation [11]:

$$
\begin{aligned}
& \Psi_{t}+3\left(\Psi^{\prime \prime}+u \Psi\right)=0, \\
& \Phi_{t}-3\left(\Phi^{\prime \prime}+u \Phi\right)=0, \\
& u_{t}+6(\Psi \Phi)^{\prime}=0 .
\end{aligned}
$$

The relation is established by the following map, which takes a solution $q, r, u_{j}$ $(j=0,1,2)$ of (3.6) (3.7), (3.8) into a solution $\Psi, \Phi, u$ of (3.9), (3.10), (3.11) and is an analog of the Miura map in the case of $\mathrm{KdV}$ and $\mathrm{mKdV}$ equations:

$$
\Psi=-q^{\prime}-q u_{0}, \quad \Phi=r^{\prime}-r u_{2}, \quad-u=u_{0}^{2}+u_{2}^{2}+u_{0} u_{2}+u_{0}^{\prime}+q r^{\prime} .
$$




\section{Similarity reduction}

In this section we consider a self-similarity condition on the solutions of the modified Yajima-Oikawa equation (3.6), (3.7), (3.8). These are the main object of this paper. A solution $q(x, t), r(x, t), u_{j}(x, t)(j=0,1,2)$ is said to be self-similar if

$$
q\left(\lambda^{2} x, \lambda^{4} t\right)=\lambda^{-1} q(x, t), \quad r\left(\lambda^{2} x, \lambda^{4} t\right)=\lambda^{-1} r(x, t), \quad u_{j}\left(\lambda^{2} x, \lambda^{4} t\right)=\lambda^{-2} u_{j}(x, t) .
$$

Here we count a degree of variables by $\operatorname{deg} x=\operatorname{deg} t_{2}=-2, \operatorname{deg} t=\operatorname{deg} t_{4}=-4$. Note that such functions are uniquely determined by its values at fixed $t$, say at $t=1 / 4$. Differentiating (4.1) with respect to $\lambda$ at $\lambda=1$, we obtain the Euler equations

$$
2 x \frac{\partial q}{\partial x}+4 t \frac{\partial q}{\partial t}=-q, \quad 2 x \frac{\partial r}{\partial x}+4 t \frac{\partial r}{\partial t}=-r, \quad 2 x \frac{\partial u_{j}}{\partial x}+4 t \frac{\partial u_{j}}{\partial t}=-2 u_{j} .
$$

At $t=1 / 4$ these identities become

$$
\frac{\partial q}{\partial t}=-2 \frac{\partial(x q)}{\partial x}+q, \quad \frac{\partial r}{\partial t}=-2 \frac{\partial(x r)}{\partial x}+r, \quad \frac{\partial u_{j}}{\partial t}=-2 \frac{\partial\left(x u_{j}\right)}{\partial x} .
$$

This can be written in the matrix form

$$
\frac{\partial B_{2}}{\partial t}=-2 \frac{\partial\left(x B_{2}\right)}{\partial x}+\left[D, B_{2}\right]
$$

where $D$ is the derivation defined in (3.1). Substituting this last identity into the zerocurvature equation (3.5), we obtain

$$
\frac{\partial M}{\partial x}=\left[4 z \frac{\partial}{\partial z}-M, B_{2}\right]
$$

where we set

$$
M=\left[\begin{array}{ccc}
\varepsilon_{1} & f_{1} & g \\
0 & \varepsilon_{2} & f_{2} \\
0 & 0 & \varepsilon_{3}
\end{array}\right]+z\left[\begin{array}{ccc}
1 & 0 & 0 \\
3 q & -2 & 0 \\
f_{0} & 3 r & 1
\end{array}\right]:=\operatorname{diag}(-1,0,1)+2 x B_{2}+B_{4} .
$$

The correspondence of variables is given as follows: $\mathrm{v}$

$$
\begin{aligned}
& \varepsilon_{1}=-1+2 x u_{0}-3 q\left(r^{\prime}-r u_{2}\right), \\
& \varepsilon_{2}=2 x u_{1}+3\left(q r^{\prime}-q^{\prime} r+q r u_{1}\right), \\
& \varepsilon_{3}=1+2 x u_{2}+3 r\left(q^{\prime}+q u_{0}\right)
\end{aligned}
$$

and $g=2 x$,

$$
f_{0}=2 x-3 q r, \quad f_{1}=2 x r+3\left(r^{\prime}-r u_{2}\right), \quad f_{2}=2 x q-3\left(q^{\prime}+q u_{0}\right) .
$$

Here we regard the variables $q=q(x, 1 / 4), r=r(x, 1 / 4), u_{j}=u_{j}(x, 1 / 4)(j=0,1,2)$ are functions only in $x$. Note that the definition of $M$ has a freedom of adding a constant diagonal matrix and here we normalize

$$
\varepsilon_{1}+\varepsilon_{2}+\varepsilon_{3}=0 .
$$




\section{Lax pair formalism}

Consider the following system of linear differential equations for the column vector $\vec{\psi}=$ ${ }^{t}\left(\psi_{1}, \psi_{2}, \psi_{3}\right)$ of three unknown functions $\psi_{i}=\psi_{i}(z, x)(i=1,2,3)$ :

$$
4 z \frac{\partial}{\partial z} \vec{\psi}=M \vec{\psi}, \quad \frac{\partial}{\partial x} \vec{\psi}=B \vec{\psi}
$$

We assume that the matrix $M$ is (4.3) and $B=B_{2}$ (3.3) where the variables $\varepsilon_{j}, f_{j}, u_{j}, q, r$ and $g$ are functions in $x$. Then the compatibility condition of system (5.1)

$$
\left[4 z \frac{\partial}{\partial z}-M, \frac{\partial}{\partial x}-B\right]=0
$$

is equivalent to the relations

$$
\begin{array}{ll}
\varepsilon_{1}^{\prime}=\varepsilon_{2}^{\prime}=\varepsilon_{3}^{\prime}=0, & g=f_{0}+3 q r \\
f_{0}^{\prime}=f_{0}\left(u_{2}-u_{0}\right)-\left(\varepsilon_{3}-\varepsilon_{1}-4\right), & g^{\prime}=g\left(u_{0}-u_{2}\right)-q f_{1}+r f_{2}-\varepsilon_{1}+\varepsilon_{3}, \\
f_{1}^{\prime}=f_{1}\left(u_{0}-u_{1}\right)-r\left(\varepsilon_{1}-\varepsilon_{2}\right), & 3 q^{\prime}=3 q\left(u_{1}-u_{0}\right)+q f_{0}-f_{2}, \\
f_{2}^{\prime}=f_{2}\left(u_{1}-u_{2}\right)-q\left(\varepsilon_{2}-\varepsilon_{3}\right), & 3 r^{\prime}=3 r\left(u_{2}-u_{1}\right)-r f_{0}+f_{1} .
\end{array}
$$

If we forget the relation (4.3) of $M$ and $B_{1}, B_{2}$ and start from the Lax equation (5.3), we can recover some of the relations of variables. For instance, differentiating both-hand side of $g=f_{0}+3 q r$ and eliminate the variables except $g^{\prime}$ by means of (5.3) , we get $g^{\prime}=2$ and therefore assume

$$
g=2 x
$$

In what follows we shall impose the following constraint on the variables:

$$
u_{0}+u_{1}+u_{2}=0, \quad u_{1}=q r .
$$

The joint system (5.2) and (5.4) is the main object that we investigate in this paper. Using system (5.3) together with the constraint, we can derive the following equation:

$$
2 g u_{0}=q f_{1}-r f_{2}-g q r-\varepsilon_{3}+\varepsilon_{1}+2 .
$$

After the elimination of the variables $f_{0}, u_{0}, u_{1}, u_{2}$ by (5.3), (5.4) and (5.5), we obtain a system of ODE for the unknown functions $f_{1}, f_{2}, q, r$ with the parameters $\varepsilon_{1}, \varepsilon_{2}, \varepsilon_{3}$. We can obtain the set of explicit formulae of $f_{1}^{\prime}, f_{2}^{\prime}, q^{\prime}, r^{\prime}$ in terms of $f_{1}, f_{2}, q, r$ and $g$, and the results are

$$
\begin{aligned}
f_{1}^{\prime} & =\frac{f_{1}}{2 g}\left(f_{1} q-f_{2} r\right)-\frac{3}{2} f_{1} q r+\left(\varepsilon_{1}-\varepsilon_{3}\right) \frac{f_{1}}{2 g}-\left(\varepsilon_{1}-\varepsilon_{2}\right) r+\frac{f_{1}}{g} \\
f_{2}^{\prime} & =\frac{f_{2}}{2 g}\left(f_{1} q-f_{2} r\right)+\frac{3}{2} f_{2} q r+\left(\varepsilon_{1}-\varepsilon_{3}\right) \frac{f_{2}}{2 g}-\left(\varepsilon_{2}-\varepsilon_{3}\right) q+\frac{f_{2}}{g} \\
q^{\prime} & =-\frac{q}{2 g}\left(f_{1} q-f_{2} r\right)+\frac{q^{2} r}{2}-\left(\varepsilon_{1}-\varepsilon_{3}\right) \frac{q}{2 g}+\frac{g q-f_{2}}{3}-\frac{q}{g} \\
r^{\prime} & =-\frac{r}{2 g}\left(f_{1} q-f_{2} r\right)-\frac{q r^{2}}{2}-\left(\varepsilon_{1}-\varepsilon_{2}\right) \frac{r}{2 g}-\frac{g r-f_{1}}{3}-\frac{r}{g} .
\end{aligned}
$$


In Sect 7 we present the system of ODE in the Hamiltonian form.

Remark. Using (5.5) and (5.3) , we can also derive the following differential equation:

$$
g u_{0}^{\prime}=\left(\varepsilon_{2}-\varepsilon_{3}\right) q r+\frac{f_{2}}{3}\left(r f_{0}-f_{1}\right)-2 u_{0} .
$$

\section{Bäcklund transformations}

Let us pass to the investigation of a group of Bäcklund transformations. For this purpose, it is convenient to introduce the following set of parameters:

$$
\alpha_{0}=\varepsilon_{3}-\varepsilon_{1}-4, \quad \alpha_{1}=\varepsilon_{1}-\varepsilon_{2}, \quad \alpha_{2}=\varepsilon_{2}-\varepsilon_{3} .
$$

They are identified with the simple roots of the affine root system of type $A_{2}^{(1)}$.

We define the Bäcklund transformations for the system by considering the gauge transformations of the linear system (5.1)

$$
s_{i} \vec{\psi}=G_{i} \vec{\psi} \quad(i=0,1,2) .
$$

The matrices $G_{i}$ are given as follows:

$$
G_{i}=1+\frac{\alpha_{i}}{f_{i}} F_{i} \quad(i=0,1,2)
$$

where $F_{0}, F_{1}, F_{2}$ are Chevalley generators (2.1) of the loop algebra $\mathfrak{s l}_{3}\left(\mathbf{C}\left[z, z^{-1}\right]\right)$. The compatibility condition of (5.1) and (6.2) is

$$
s_{i}(M)=G_{i} M G_{i}^{-1}+4 z \frac{\partial G_{i}}{\partial z} G_{i}^{-1}, \quad s_{i}(B)=G_{i} B G_{i}^{-1}+\frac{\partial G_{i}}{\partial x} G_{i}^{-1} .
$$

On the components of the matrices $M, B$, the actions of $s_{i}(i=0,1,2)$ are given explicitly as in the following tables:

\begin{tabular}{c|ccc|ccc} 
& $f_{0}$ & $f_{1}$ & $f_{2}$ & $g$ & $q$ & $r$ \\
\hline$s_{0}$ & $f_{0}$ & $f_{1}+3 r \frac{\alpha_{0}}{f_{0}}$ & $f_{2}-3 q \frac{\alpha_{0}}{f_{0}}$ & $g$ & $q$ & $r$ \\
$s_{1}$ & $f_{0}-3 r \frac{\alpha_{1}}{f_{1}}$ & $f_{1}$ & $f_{2}+g \frac{\alpha_{1}}{f_{1}}$ & $g$ & $q+\frac{\alpha_{1}}{f_{1}}$ & $r$ \\
$s_{2}$ & $f_{0}+3 q \frac{\alpha_{2}}{f_{2}}$ & $f_{1}-g \frac{\alpha_{2}}{f_{2}}$ & $f_{2}$ & $g$ & $q$ & $r-\frac{\alpha_{2}}{f_{2}}$
\end{tabular}

\begin{tabular}{c|ccc|ccc} 
& $\alpha_{0}$ & $\alpha_{1}$ & $\alpha_{2}$ & $u_{0}$ & $u_{1}$ & $u_{2}$ \\
\hline$s_{0}$ & $-\alpha_{0}$ & $\alpha_{1}+\alpha_{0}$ & $\alpha_{2}+\alpha_{0}$ & $u_{0}+\frac{\alpha_{0}}{f_{0}}$ & $u_{1}$ & $u_{2}-\frac{\alpha_{0}}{f_{0}}$ \\
$s_{1}$ & $\alpha_{0}+\alpha_{1}$ & $-\alpha_{1}$ & $\alpha_{2}+\alpha_{1}$ & $u_{0}-r \frac{\alpha_{1}}{f_{1}}$ & $u_{1}+r \frac{\alpha_{1}}{f_{1}}$ & $u_{2}$ \\
$s_{2}$ & $\alpha_{0}+\alpha_{2}$ & $\alpha_{1}+\alpha_{2}$ & $-\alpha_{2}$ & $u_{0}$ & $u_{1}-q \frac{\alpha_{2}}{f_{2}}$ & $u_{2}+q \frac{\alpha_{2}}{f_{2}}$
\end{tabular}


The automorphisms $s_{i}(i=0,1,2)$ generate a group of Bäcklund transformations for our differential system. To state this fact clearly, it is convenient to introduce the field

$$
K=\mathbf{C}\left(\alpha_{0}, \alpha_{1}, \alpha_{2}, f_{0}, f_{1}, f_{2}, g, q, r, u_{0}, u_{1}, u_{2}\right),
$$

where the generators satisfy the following algebraic relations:

$$
\begin{gathered}
\alpha_{0}+\alpha_{1}+\alpha_{2}=-4, \quad f_{0}=g-3 q r, \quad u_{0}+u_{1}+u_{2}=0, \quad u_{1}=q r, \\
2 g u_{0}=q f_{1}-r f_{2}-g q r-\varepsilon_{3}+\varepsilon_{1}+2 .
\end{gathered}
$$

We have the automorphisms $s_{i}(i=0,1,2)$ of the field $K$ defined by the above table. Note that the field $K$ is thought to be a differential field with the derivation ' $: K \rightarrow K$ defined by (5.3).

Theorem 1 The automorphism $s_{0}, s_{1}, s_{2}$ of $K$ define a representation of the affine Weyl group $W$ (2.2) on the field $K$ such that the action of the each element $w \in W$ commutes with the derivation of the differential field $K$.

Theorem 10 is proved by straightforward computations. Note that the independent variable $x=g / 2$ is fixed under the action of $W$.

\section{Hamiltonian structure}

We shall equip $K$ (6.5) with the Poisson algebra structure $\{\}:, K \times K \rightarrow K$ defined as follows:

\begin{tabular}{c|cccc}
$\{\}$, & $f_{1}$ & $f_{2}$ & $q$ & $r$ \\
\hline$f_{1}$ & 0 & $g$ & 1 & 0 \\
$f_{2}$ & $-g$ & 0 & 0 & -1 \\
$q$ & -1 & 0 & 0 & 0 \\
$r$ & 0 & 1 & 0 & 0
\end{tabular}

That is, $\left\{f_{1}, f_{2}\right\}=g$ and so on. Note that the Poisson structure comes from the Lie algebra structure of $\hat{\mathfrak{g}}$ (see [9] for an exposition). We can describe the action of $s_{i}(i=0,1,2)$ on the generators $f=f_{j}, u_{j}, q, r, g(j=0,1,2)$ of $K$ by

$$
s_{i}(f)=f+\frac{\alpha_{i}}{f_{i}}\left\{f_{i}, f\right\} .
$$

We introduce the function $h$ by

$$
\begin{aligned}
h:= & \frac{1}{2}\left(f_{1} q^{2} r+f_{2} q r^{2}\right)-\frac{1}{4 g}\left(f_{1}^{2} q^{2}+f_{2}^{2} r^{2}+q^{2} r^{2} g^{2}\right)+\left(\frac{q r}{2 g}-\frac{1}{3}\right) f_{1} f_{2} \\
& +\left(\frac{g}{3}-\frac{\alpha_{1}+\alpha_{2}}{2 g}\right) f_{1} q+\left(\frac{g}{3}+\frac{\alpha_{1}+\alpha_{2}}{2 g}\right) f_{2} r-\left(\frac{g}{3}-\frac{\alpha_{1}-\alpha_{2}}{2 g}\right) q r g .
\end{aligned}
$$


Then the differential system (5.6) -(5.9) can be expressed

$$
\begin{aligned}
& f_{1}^{\prime}=\left\{h, f_{1}\right\}+\frac{f_{1}}{g}, \quad q^{\prime}=\{h, q\}-\frac{q}{g}, \\
& f_{2}^{\prime}=\left\{h, f_{2}\right\}+\frac{f_{2}}{g}, \quad r^{\prime}=\{h, r\}-\frac{r}{g} .
\end{aligned}
$$

Let us introduce the variables

$$
p_{1}=f_{1}, \quad q_{1}=q, \quad p_{2}=\frac{f_{2}}{g}-q, \quad q_{2}=-g r .
$$

It is easy to show that

$$
\left\{p_{i}, q_{j}\right\}=\delta_{i j}, \quad\left\{p_{i}, p_{j}\right\}=\left\{q_{i}, q_{j}\right\}=0 \quad(i, j=1,2) .
$$

Theorem 2 Let $H$ be the function defined as

$$
\begin{aligned}
x H= & -\frac{1}{4} p_{1} p_{2} q_{1} q_{2}-\frac{1}{8}\left(p_{1}^{2} q_{1}^{2}+p_{2}^{2} q_{2}^{2}\right)-\frac{1}{2} p_{1} q_{1}^{2} q_{2} \\
& -\frac{1}{4}\left(\alpha_{1}+\alpha_{2}+2\right) p_{1} q_{1}-\frac{1}{4}\left(\alpha_{1}+\alpha_{2}-2\right) p_{2} q_{2}-\frac{\alpha_{1}}{2} q_{1} q_{2}-\frac{2 x^{2}}{3}\left(q_{2}+p_{1}\right) p_{2}
\end{aligned}
$$

Then the system of ODEs (5.6), (5.7), (5.8), (5.9) is equivalent to the Hamiltonian system

$$
\frac{d q_{1}}{d x}=\frac{\partial H}{\partial p_{1}}, \quad \frac{d q_{2}}{d x}=\frac{\partial H}{\partial p_{2}}, \quad \frac{d p_{1}}{d x}=-\frac{\partial H}{\partial q_{1}}, \quad \frac{d p_{2}}{d x}=-\frac{\partial H}{\partial q_{2}} .
$$

Proof. We define

$$
H=h-\frac{f_{1} q+f_{2} r}{g}+q r
$$

and rewrite this in the coordinate $p_{j}, q_{j}(j=1,2)$. Then the equations (7.1) can be expressed as (7.2).

The behavior of the Hamiltonian under the Bäcklund transformations is given by the simple formulae

$$
s_{0}(\tilde{H})=\tilde{H}+6 q r \frac{\alpha_{0}}{f_{0}}, \quad s_{j}(\tilde{H})=\tilde{H}(j=1,2),
$$

where we set $\tilde{H}=x H+a$ with the correction term

$$
a=\frac{1}{24}\left(\alpha_{1}-\alpha_{2}\right)\left(\alpha_{1}-\alpha_{2}-4\right)
$$




\section{Reduction to the fifth Painlevé equation}

In this section, we show the system (5.3) is equivalent to a two-parameter family of the fifth Painlevé equation. By linear change of the independent variable, we ensure the normalization

$$
f_{0}+\frac{f_{1}}{r}+\frac{f_{2}}{q}+3\left(\frac{q^{\prime}}{q}-\frac{r^{\prime}}{r}\right)=3 g=6 x
$$

holds. After the elimination of $u_{0}$ and $u_{2}$, we have

$$
\begin{aligned}
f_{0}^{\prime} & =-\frac{f_{0}}{3}\left(\frac{f_{1}}{r}-\frac{f_{2}}{q}\right)+\frac{f_{0} u_{1}^{\prime}}{u_{1}}-\alpha_{0} \\
\left(\frac{f_{1}}{r}\right)^{\prime} & =-\frac{f_{1}}{3 r}\left(\frac{f_{2}}{q}-f_{0}+\frac{3 u_{1}^{\prime}}{u_{1}}\right)-\alpha_{1}, \\
\left(\frac{f_{2}}{q}\right)^{\prime} & =-\frac{f_{2}}{3 q}\left(f_{0}-\frac{f_{1}}{r}+\frac{3 u_{1}^{\prime}}{u_{1}}\right)-\alpha_{2} .
\end{aligned}
$$

Here we introduce a new variable

$$
y:=-\frac{f_{0}}{3 u_{1}}
$$

Notice the relations

$$
y-1=-\frac{2 x}{3 u_{1}}, \quad \frac{y^{\prime}}{y-1}=\frac{1}{x}-\frac{u_{1}^{\prime}}{u_{1}}
$$

holds by $f_{0}=g-3 q r=2 x-3 u_{1}$. Then we rewrite (8.2) as

$$
y^{\prime}=-\frac{y}{3}\left(\frac{f_{1}}{r}-\frac{f_{2}}{q}\right)+\frac{\alpha_{0}}{3 u_{1}}
$$

After differentiating (8.6), elimination of the variables $f_{1}, f_{2}, q, r, u_{1}$ by (8.1), (8.3), (8.4), (8.5), (8.6) and the definition of the constant $\varepsilon_{2}$ (4.5) leads to the following equation of $y$ :

$$
\begin{gathered}
y^{\prime \prime}=\left(\frac{1}{2 y}+\frac{1}{y-1}\right)\left(y^{\prime}\right)^{2}-\frac{y^{\prime}}{x}+\frac{(y-1)^{2}}{8 x^{2}}\left(\varepsilon_{2}^{2} y-\frac{\alpha_{0}^{2}}{y}\right) \\
-\frac{2 x^{2} y}{9}-\frac{4 x^{2} y}{9(y-1)}-\frac{\left(\alpha_{2}-\alpha_{1}\right) y}{3}+\frac{\varepsilon_{2} y}{3}
\end{gathered}
$$

We put $\xi=x^{2}$, then the equation (8.7) can be brought into the fifth Painlevé equation

$$
y_{\xi \xi}=\left(\frac{1}{2 y}+\frac{1}{y-1}\right)\left(y_{\xi}\right)^{2}-\frac{1}{\xi} y_{\xi}+\frac{(y-1)^{2}}{\xi^{2}}\left(A y+\frac{B}{y}\right)+\frac{C}{\xi} y-\frac{y(y+1)}{18(y-1)},
$$

where

$$
A=\frac{\varepsilon_{2}^{2}}{32}, \quad B=-\frac{\alpha_{0}^{2}}{32}, \quad C=-\frac{\varepsilon_{2}}{6} .
$$

Note that $\varepsilon_{2}=\left(\alpha_{2}-\alpha_{1}\right) / 3$ holds by (4.8) and (6.1). 


\section{$9 \tau$-functions}

We introduce the $\tau$-functions $\tau_{0}, \tau_{1}, \tau_{2}, \sigma_{1}$ and $\sigma_{2}$ to be the dependent variables satisfying the following equations:

$$
\frac{f_{1}}{r}=2 x+3\left(\frac{\sigma_{2}^{\prime}}{\sigma_{2}}-\frac{\tau_{0}^{\prime}}{\tau_{0}}\right), \quad \frac{f_{2}}{q}=2 x-3\left(\frac{\sigma_{1}^{\prime}}{\sigma_{1}}-\frac{\tau_{0}^{\prime}}{\tau_{0}}\right), \quad q=-\frac{\sigma_{1}}{\tau_{1}}, \quad r=\frac{\sigma_{2}}{\tau_{2}} .
$$

To fix the freedom of overall multiplication by a function in the defining equation (9.1) for $\tau_{0}, \tau_{1}, \tau_{2}, \sigma_{1}$ and $\sigma_{2}$, we impose the equation

$$
\begin{aligned}
\left(\log \tau_{0}^{2} \tau_{1}^{2} \tau_{2}^{2} \sigma_{1} \sigma_{2}\right)^{\prime \prime} & +u_{0}^{2}+u_{2}^{2}+\left(u_{0}-\frac{f_{1}}{3 r}+\frac{2 x}{3}\right)^{2}+\left(u_{2}-\frac{f_{2}}{3 q}+\frac{2 x}{3}\right)^{2} \\
& -\frac{2 x}{9}\left(4 x-\frac{f_{1}}{r}-\frac{f_{2}}{q}\right)-\frac{\alpha_{1}-\alpha_{2}}{9}=0 .
\end{aligned}
$$

The differential equations for the variables $q$ and $r$ in the system (5.3) lead to

$$
u_{0}=\frac{\tau_{1}^{\prime}}{\tau_{1}}-\frac{\tau_{0}^{\prime}}{\tau_{0}}, \quad u_{2}=\frac{\tau_{0}^{\prime}}{\tau_{0}}-\frac{\tau_{2}^{\prime}}{\tau_{2}}
$$

respectively. Here we have used the relations

$$
u_{1}=q r=-\frac{\sigma_{1} \sigma_{2}}{\tau_{1} \tau_{2}}, \quad f_{0}=2 x-3 q r=2 x+3 \frac{\sigma_{1} \sigma_{2}}{\tau_{1} \tau_{2}} .
$$

If the equations (9.3) are satisfied, we have

$$
u_{1}=\frac{\tau_{2}^{\prime}}{\tau_{2}}-\frac{\tau_{1}^{\prime}}{\tau_{1}}
$$

by $u_{0}+u_{1}+u_{2}=0$ and therefore have the following formula of the variable $f_{0}$ in terms of the $\tau$-functions:

$$
f_{0}=2 x+3\left(\frac{\tau_{1}^{\prime}}{\tau_{1}}-\frac{\tau_{2}^{\prime}}{\tau_{2}}\right) .
$$

Let $D_{x}$ and $D_{x}^{2}$ be Hirota's bilinear operators:

$$
D_{x} F \cdot G:=F^{\prime} G-F G^{\prime}, \quad D_{x}^{2} F \cdot G:=F^{\prime \prime} G-2 F^{\prime} G^{\prime}+F G^{\prime \prime} .
$$

In this notation, the relation $u_{1}=q r$, for example, can be written in

$$
D_{x} \tau_{1} \cdot \tau_{2}=\sigma_{1} \sigma_{2}
$$

We introduce a system of bilinear equations that leads to our differential system (5.3). 
Theorem 3 Let $\tau_{0}, \tau_{1}, \tau_{2}, \sigma_{1}, \sigma_{2}$ be a set of functions that satisfies the following system of Hirota bilinear equations:

$$
\begin{aligned}
& \left(3 D_{x}^{2}-2 x D_{x}+\frac{1}{6}\left(\alpha_{0}-4 \alpha_{1}-2\right)\right) \tau_{0} \cdot \tau_{1}=0 \\
& \left(3 D_{x}^{2}-2 x D_{x}-\frac{1}{6}\left(\alpha_{0}-4 \alpha_{2}-2\right)\right) \tau_{2} \cdot \tau_{0}=0 \\
& \left(3 D_{x}^{2}-2 x D_{x}+\frac{1}{6}\left(\alpha_{1}-\alpha_{2}+6\right)\right) \tau_{1} \cdot \sigma_{2}=0 \\
& \left(3 D_{x}^{2}-2 x D_{x}+\frac{1}{6}\left(\alpha_{1}-\alpha_{2}-6\right)\right) \sigma_{1} \cdot \tau_{2}=0
\end{aligned}
$$

together with (9.6). If we define the functions $f_{0}, f_{1}, f_{2}, q, r, u_{0}, u_{1}$ and $u_{2}$ by the formulae (9.1), (9.3), (9.4) then this set of functions satisfies our ODE system (5.3) together with algebraic equations (5.4).

Proof. We can verify that the differential equations for $q$ and $r$ are satisfied if we assume the existence of the $\tau$-functions such that equations (9.1), (9.3) holds. The differential equations for $f_{0}$ is written as

$$
3\left(g_{1}^{\prime \prime}-g_{2}^{\prime \prime}\right)+2=\left(3\left(g_{1}^{\prime}-g_{2}^{\prime}\right)+2 x\right)\left(2 g_{0}^{\prime}-g_{1}^{\prime}-g_{2}^{\prime}\right)-\alpha_{0}
$$

where $g_{j}=\log \tau_{j},(j=0,1,2)$. This equation is obtained if we subtract (9.7) from (9.8). The differential equations for $f_{1}$ and $f_{2}$ can be rewritten as

$$
\left(\frac{f_{1}}{r}\right)^{\prime}=\frac{f_{1}}{r}\left(u_{0}-u_{1}-\frac{r^{\prime}}{r}\right)-\alpha_{1}\left(\frac{f_{2}}{q}\right)^{\prime}=\frac{f_{2}}{q}\left(u_{1}-u_{2}-\frac{q^{\prime}}{q}\right)-\alpha_{2},
$$

respectively. In terms of the $\tau$-functions, these equations read

$$
\begin{aligned}
& 3\left(h_{2}^{\prime \prime}-g_{0}^{\prime \prime}\right)+2=\left(3\left(h_{2}^{\prime}-g_{0}^{\prime}\right)+2 x\right)\left(2 g_{1}^{\prime}-g_{0}^{\prime}-h_{2}^{\prime}\right)-\alpha_{1}, \\
& 3\left(g_{0}^{\prime \prime}-h_{1}^{\prime \prime}\right)+2=\left(3\left(g_{0}^{\prime}-h_{1}^{\prime}\right)+2 x\right)\left(2 g_{2}^{\prime}-g_{0}^{\prime}-g_{1}^{\prime}\right)-\alpha_{2},
\end{aligned}
$$

where $h_{1}=\log \sigma_{1}, h_{2}=\log \sigma_{2}$. In fact, from (9.7) and (9.9) we can eliminate $g_{1}^{\prime \prime}$ to obtain (9.13). In the similar way from (9.8) and (9.10), we can eliminate $g_{2}^{\prime \prime}$ to obtain (9.14).

We remark that the normalization of $\tau$-functions (9.2) is obtained by taking the sum of four equations in this theorem.

\section{Jacobi-Trudi type formula}

In this section we lift the action of $W$ to the $\tau$-functions. Consider the field extension $\widetilde{K}=K\left(\tau_{0}, \tau_{1}, \tau_{2}, \sigma_{1}, \sigma_{2}\right)$. Then we can prove the next Theorem by a direct computation. 
Theorem 4 We extend each automorphism $s_{i}$ of $K$ to an automorphism of the field $\widetilde{K}=K\left(\tau_{0}, \tau_{1}, \tau_{2}, \sigma_{1}, \sigma_{2}\right)$ by the formulae $s_{i}\left(\tau_{j}\right)=\tau_{j}(i \neq j), s_{i}\left(\sigma_{k}\right)=\sigma_{k}(i \neq k)$ and

$$
\begin{array}{ll}
s_{0}\left(\tau_{0}\right)=f_{0} \frac{\tau_{2} \tau_{1}}{\tau_{0}}, & s_{1}\left(\tau_{1}\right)=f_{1} \frac{\tau_{0} \tau_{2}}{\tau_{1}}, \quad s_{1}\left(\sigma_{1}\right)=-\left(f_{1} q+\alpha_{1}\right) \frac{\tau_{0} \tau_{2}}{\tau_{1}}, \\
s_{2}\left(\tau_{2}\right)=f_{2} \frac{\tau_{1} \tau_{0}}{\tau_{2}}, & s_{2}\left(\sigma_{2}\right)=\left(f_{2} r-\alpha_{2}\right) \frac{\tau_{1} \tau_{0}}{\tau_{2}} .
\end{array}
$$

Then these automorphisms define a representation of $W$ on $\widetilde{K}$.

Following [6], we will describe the Weyl group orbit of the $\tau$-functions (see also 9]). For any $w \in W$ and $k=0,1,2$, there exists a rational function $\phi_{w}^{(k)} \in K$ such that

$$
w\left(\tau_{k}\right)=\phi_{w}^{(k)} \prod_{i=0,1,2} \tau_{i}^{\left(\alpha_{i} \mid w\left(\Lambda_{k}\right)\right)} .
$$

We shall give an expression of $\phi_{w}^{(k)}$ in terms of the Jacobi-Trudi type determinant.

A subset $M$ of $\mathbf{Z}$ is called a Maya diagram if $M \cap \mathbf{Z}_{\geq 0}$ and $M^{c} \cap \mathbf{Z}_{<0}$ are finite sets. We define an integer

$$
c(M):=\sharp\left(M \cap \mathbf{Z}_{\geq 0}\right)-\sharp\left(M^{c} \cap \mathbf{Z}_{<0}\right)
$$

called the charge of $M$. If $c(M)=r$, we can express $M$ as $\left\{i_{k} \mid k<r\right\}$ by using an strictly increasing sequence $i_{k}(k<r)$ such that $i_{k}=k$ for $k \ll r$. Then we associate a partition $\lambda=\left(\lambda_{1}, \lambda_{2}, \ldots\right)$ given by

$$
\lambda_{j}=i_{r-j+1}-(r-j+1), \quad(j=1,2, \ldots) .
$$

The Weyl group $W=\left\langle s_{0}, s_{1}, s_{2}\right\rangle$ can be realized as a subgroup of the group of bijections $w: \mathbf{Z} \rightarrow \mathbf{Z}$ by setting

$$
s_{k}=\prod_{j \in \mathbf{Z}} \sigma_{3 j+k-1} \quad(k=0,1,2),
$$

where $\sigma_{i}(i \in \mathbf{Z})$ is the adjacent transposition $(i, i+1)$. For a Maya diagram $M$ and $w \in W$, we see that $w(M) \subset \mathbf{Z}$ is also a Maya diagram of the same charge.

For any $w \in W$ and $k=0,1,2$, let $\lambda=\left(\lambda_{1}, \ldots, \lambda_{r}\right)$ be the partition corresponding to the Maya diagram $M=w\left(\mathbf{Z}_{<k}\right)$. We set

$$
N_{\lambda}^{(k)}=\prod_{\substack{i<j \\ i \in M^{c}, j \in M}}\left(\varepsilon_{i}-\varepsilon_{j}\right),
$$

where we impose the relation $\varepsilon_{i}-\varepsilon_{i+3}=-4(i \in \mathbf{Z})$, so we have $N_{\lambda}^{(k)} \in \mathbf{C}\left[\alpha_{0}, \alpha_{1}, \alpha_{2}\right]$. We can apply the following formula due to Y.Yamada [12]:

$$
\phi_{w}\left(\Lambda_{k}\right)=N_{\lambda}^{(k)} \operatorname{det}\left(g_{\lambda_{j}-j+i}^{(k-i+1)}\right)_{1 \leq i, j \leq r} .
$$


Here $g_{p}^{(k)}\left(k \in \mathbf{Z} / 3 \mathbf{Z}, p \in \mathbf{Z}_{>0}\right)$ are the determinant of $p \times p$ matrix described as follows. First we define $g_{p}^{(0)}$ by

$$
g_{p}^{(0)}:=\frac{1}{N_{p}^{(0)}}\left|\begin{array}{cccccc}
f_{00} & f_{01} & f_{02} & & & 0 \\
\beta_{1} & f_{11} & f_{12} & f_{13} & & \\
& \ddots & \ddots & \ddots & \ddots & \\
& & \ddots & \ddots & \ddots & f_{p-3, p-1} \\
& & & \beta_{p-2} & f_{p-2, p-2} & f_{p-2, p-1} \\
0 & & & & \beta_{p-1} & f_{p-1, p-1}
\end{array}\right|,
$$

where the components are

$$
\begin{aligned}
f_{i, i} & =f_{i} \quad\left(f_{i+3}=f_{i}\right), \\
f_{i, i+1} & =g(i \equiv 1), \quad 3 q(i \equiv 2), \quad 3 r(i \equiv 0), \\
f_{i, i+2} & =1(i \equiv 1,0), \quad-2(i \equiv 2),
\end{aligned}
$$

and $\beta_{j}=\sum_{i=j}^{p-1} \alpha_{i}=\varepsilon_{j}-\varepsilon_{p}$. Then we put $g_{p}^{(1)}=\pi\left(g_{p}^{(0)}\right)$ and $g_{p}^{(2)}=\pi^{2}\left(g_{p}^{(0)}\right)$ by the automorphism $\pi$ :

$$
\pi\left(f_{i j}\right)=f_{i+1, j+1}, \quad \pi\left(\varepsilon_{j}\right)=\varepsilon_{j+1} .
$$

The formula (10.4) is valid since the action of $W=\left\langle s_{0}, s_{1}, s_{2}\right\rangle$ in our setting is reduced from the action of $A_{\infty}$ (cf. [9]):

$$
s_{i}\left(\alpha_{i}\right)=-\alpha_{i}, \quad s_{i}\left(\alpha_{i \pm 1}\right)=\alpha_{i \pm 1}+\alpha_{i}, \quad s_{i}\left(\alpha_{j}\right)=\alpha_{j}(j \neq i, i \pm 1),
$$

where $\alpha_{j}:=\varepsilon_{j}-\varepsilon_{j+1}(j \in \mathbf{Z})$ and

$$
s_{k}\left(f_{i, j}\right)=f_{i, j}+\left(\delta_{k+1, i} f_{k, j}-\delta_{j, k} f_{i, k+1}\right) \frac{\alpha_{k}}{f_{k}} .
$$

\section{Differential field of $\tau$-functions}

In this section we give supplementary discussions on the affine Weyl group action. In particular, we consider a differential field of $\tau$-functions that naturally contains the fields $K$ and $\widetilde{K}$. The field $\widehat{F}$ we consider can be presented as

$$
\mathbf{C}\left(\alpha_{0}, \alpha_{1}, \alpha_{2}, x ; \tau_{0}, \tau_{1}, \tau_{2}, \sigma_{1}, \sigma_{2}, \tau_{0}^{\prime}, \tau_{1}^{\prime}, \tau_{2}^{\prime}, \sigma_{1}^{\prime}, \sigma_{2}^{\prime}\right)
$$

with some relations discussed below. Then the set of bilinear equations in Theorem 3 makes $\widehat{F}$ into the differential field. To show some basic facts on $\widehat{F}$, we introduce some intermediate fields.

Let $F$ denote the extended field of $\mathbf{C}\left(\alpha_{0}, \alpha_{1}, \alpha_{2}, x\right)$ obtained by adjoining the variables $g_{0}^{\prime}, g_{1}^{\prime}, g_{2}^{\prime}, h_{1}^{\prime}, h_{2}^{\prime}$ with the following relations:

$$
\begin{aligned}
& 3\left(g_{0}^{\prime}-2 h_{2}^{\prime}+h_{1}^{\prime}\right)\left(g_{1}^{\prime}-g_{2}^{\prime}\right)+2 x\left(g_{0}^{\prime}-2 g_{1}^{\prime}+g_{2}^{\prime}\right)+\alpha_{1}+1=0, \\
& 3\left(h_{2}^{\prime}-2 h_{1}^{\prime}+g_{0}^{\prime}\right)\left(g_{1}^{\prime}-g_{2}^{\prime}\right)+2 x\left(g_{1}^{\prime}-2 g_{2}^{\prime}+g_{0}^{\prime}\right)+\alpha_{2}+1=0 .
\end{aligned}
$$


As in the proof of Theorem 3 , we will identify $g_{j}^{\prime}$ with $\left(\log \tau_{j}\right)^{\prime}$ and $h_{1}^{\prime}, h_{2}^{\prime}$ with $\left(\log \sigma_{1}\right)^{\prime}$, $\left(\log \sigma_{2}\right)^{\prime}$ respectively. Note that the relations (11.2), (11.3) correspond to (4.4), (4.5), (4.6). It is easy to see $F=\mathbf{C}\left(\alpha_{0}, \alpha_{1}, \alpha_{2}, x\right)\left(g_{0}^{\prime}, g_{1}^{\prime}, g_{2}^{\prime}\right)$, and $g_{0}^{\prime}, g_{1}^{\prime}, g_{2}^{\prime}$ are algebraically independent over $\mathbf{C}\left(\alpha_{0}, \alpha_{1}, \alpha_{2}, x\right)$. So if we fix $g_{j}^{\prime \prime} \in F(j=0,1,2)$ in an arbitrary way, then we have a derivation on $F$. Now we want to introduce a derivation on $F$ in such a way that is consistent with the bilinear equations. Actually we can prove the following lemma by lengthy but straightforward computations:

Lemma 1 There exists a unique derivation on $F$ such that the set of bilinear equations in Theorem 3 holds.

Consider the extended field $\widehat{F}:=F\left(\tau_{0}, \tau_{1}, \tau_{2}, \sigma_{1}, \sigma_{2}\right)$ with a relation

$$
\tau_{1}^{\prime} \tau_{2}-\tau_{2} \tau_{1}^{\prime}=\sigma_{1} \sigma_{2}
$$

We can naturally extend the derivation by $\tau_{j}^{\prime}=g_{j}^{\prime} \tau_{j}, \sigma_{k}^{\prime}=h_{k}^{\prime} \sigma_{k}(j=0,1,2, k=1,2)$. Then we have the previous presentation (11.1). Now the next lemma is a direct consequence of Theorem 3.

Lemma 2 We have a natural embedding of the differential fields

$$
K \subset \widehat{F} \text {. }
$$

Our next task is to extend the affine Weyl group action on $\widetilde{K}=K\left(\tau_{0}, \tau_{1}, \tau_{2}, \sigma_{1}, \sigma_{2}\right)$ (Theorem 4) to $\widehat{F}$. The following two lemmas can be easily verified.

Lemma 3 By the following formulae, we can introduce an action of the affine Weyl group $W$ on $\widehat{F}$ as a group of automorphisms:

$$
\begin{aligned}
& \frac{s_{0}\left(\tau_{0}^{\prime}\right)}{s_{0}\left(\tau_{0}\right)}=\frac{\tau_{0}^{\prime}}{\tau_{0}}-\frac{\alpha_{0}}{f_{0}}, \\
& \frac{s_{1}\left(\tau_{1}^{\prime}\right)}{s_{1}\left(\tau_{1}\right)}=\frac{\tau_{1}^{\prime}}{\tau_{1}}-\frac{\alpha_{1}}{f_{1}} \frac{\sigma_{2}}{\tau_{2}}, \quad \frac{s_{1}\left(\sigma_{1}^{\prime}\right)}{s_{1}\left(\tau_{1}\right)}=\frac{\sigma_{1}^{\prime}}{\tau_{1}}-\frac{\alpha_{1}}{f_{1}} \frac{\tau_{0}^{\prime}}{\tau_{0}}, \\
& \frac{s_{2}\left(\tau_{2}^{\prime}\right)}{s_{2}\left(\tau_{2}\right)}=\frac{\tau_{2}^{\prime}}{\tau_{2}}+\frac{\alpha_{2}}{f_{2}} \frac{\sigma_{1}}{\tau_{1}}, \quad \frac{s_{2}\left(\sigma_{2}^{\prime}\right)}{s_{2}\left(\tau_{2}\right)}=\frac{\sigma_{2}^{\prime}}{\tau_{2}}-\frac{\alpha_{1}}{f_{1}} \frac{\tau_{0}^{\prime}}{\tau_{0}},
\end{aligned}
$$

and $s_{i}\left(\tau_{j}^{\prime}\right)=\tau_{j}^{\prime}(i \neq j), s_{i}\left(\sigma_{k}^{\prime}\right)=\sigma_{k}^{\prime}(i \neq k)$. Moreover this action is an extension of the action of $W$ on $\widetilde{K}$.

Lemma 4 For $i, j=0,1,2$ and $k=1,2$ we have

$$
s_{i}\left(\tau_{j}^{\prime}\right)=s_{i}\left(\tau_{j}\right)^{\prime}, \quad s_{i}\left(\sigma_{k}^{\prime}\right)=s_{i}\left(\sigma_{k}\right)^{\prime} .
$$

Remark. Although we have introduced the Weyl group action on the $\tau$-functions in an ad hoc manner, these formulae can be derived systematically by using the gauge matrices $G_{i}(6.3)$, if we identify the $\tau$-functions with the components of a dressing matrix. We will give an explanation of this point in a separate article.

The goal of this section is the following fact: 
Theorem 5 The derivation of $\widehat{F}$ commutes with the action of $W$ on $\widehat{F}$.

A straightforward verification of this fact may require quite a bit of calculations, because the second derivatives of $\tau$-functions are determined implicitly by the bilinear equations. To avoid the complexity, we make use of the fact $\widehat{F}=\widetilde{K}(k)$, which is easily seen from (9.1), (9.3), and (9.4), where we set

$$
k=2\left(\frac{\tau_{0}^{\prime}}{\tau_{0}}+\frac{\tau_{1}^{\prime}}{\tau_{1}}+\frac{\tau_{2}^{\prime}}{\tau_{2}}\right)+\frac{\sigma_{1}^{\prime}}{\sigma_{1}}+\frac{\sigma_{2}^{\prime}}{\sigma_{2}} .
$$

As for the first derivatives of $\tau$-functions, we have already lemma 4, Therefore, in order to prove Theorem 5, it suffices to show the next lemma.

\section{Lemma 5}

$$
s_{i}\left(k^{\prime}\right)=s_{i}(k)^{\prime} \quad(i=0,1,2) .
$$

Proof. By Lemma 3, we have

$$
\begin{aligned}
& s_{0}(k)-k=2\left(\frac{s_{0}\left(\tau_{0}^{\prime}\right)}{s_{0}\left(\tau_{0}\right)}-\frac{\tau_{0}^{\prime}}{\tau_{0}}\right)=-2 \frac{\alpha_{0}}{f_{0}}, \\
& s_{1}(k)-k=2\left(\frac{s_{1}\left(\tau_{1}^{\prime}\right)}{s_{1}\left(\tau_{1}\right)}-\frac{\tau_{1}^{\prime}}{\tau_{1}}\right)+\left(\frac{s_{1}\left(\sigma_{1}^{\prime}\right)}{s_{1}\left(\sigma_{1}\right)}-\frac{\sigma_{1}^{\prime}}{\sigma_{1}}\right), \\
& s_{2}(k)-k=2\left(\frac{s_{2}\left(\tau_{2}^{\prime}\right)}{s_{2}\left(\tau_{2}\right)}-\frac{\tau_{2}^{\prime}}{\tau_{2}}\right)+\left(\frac{s_{2}\left(\sigma_{2}^{\prime}\right)}{s_{2}\left(\sigma_{2}\right)}-\frac{\sigma_{1}^{\prime}}{\sigma_{1}}\right) .
\end{aligned}
$$

We can rewrite the right hand sides of (11.5) and (11.6) into

$$
\begin{aligned}
& s_{1}(k)-k=-2 \frac{\alpha_{1}}{f_{1}} r-\frac{\alpha_{1}\left(2 x q-f_{2}\right)}{3 q\left(f_{1} q+\alpha_{1}\right)}, \\
& s_{2}(k)-k=-2 \frac{\alpha_{2}}{f_{2}} q-\frac{\alpha_{2}\left(2 x r-f_{1}\right)}{3 r\left(f_{2} r-\alpha_{2}\right)}
\end{aligned}
$$

by using (9.1), (10.1) and (10.2). On the other hand, the normalization condition (9.2) reads

$$
\begin{aligned}
k^{\prime}=- & u_{0}^{2}-u_{2}^{2}-\left(u_{0}-\frac{f_{1}}{3 r}+\frac{2 x}{3}\right)^{2}-\left(u_{2}-\frac{f_{2}}{3 q}+\frac{2 x}{3}\right)^{2} \\
+ & \frac{2 x}{9}\left(4 x-\frac{f_{1}}{r}-\frac{f_{2}}{q}\right)+\frac{\alpha_{1}-\alpha_{2}}{9} .
\end{aligned}
$$

Then we can verify (11.4) by applying (6.4) to $s_{i}\left(k^{\prime}\right)$ and the ODE (15.3) to $s_{i}(k)^{\prime}$. 


\section{Discussion}

We have derived a two-parameter family of the fifth Painlevé equation as a similarity reduction of the modified Yajima-Oikawa hierarchy, which is related to a non-standard Heisenberg subalgebra of $A_{2}^{(1)}$. The system admits a group of Bäcklund transformations of type $W\left(A_{2}^{(1)}\right)$. By a suitable modification of our construction, it may be possible to recover a missing parameter and get the fifth Painlevé with the full symmetry of type $W\left(A_{3}^{(1)}\right)$. Combinatorial and/or representation theoretical structure of the hierarchy is also deserves to be investigated. A combinatorial aspect of representation associated with the Yajima-Oikawa hierarchy is studied by S. Leidwanger in [5]. It seems that the work is closely related some family of polynomial solutions of the fifth Painlevé equation. We hope that we discuss these issues in future publications.

\section{Acknowledgments}

The authors are grateful to Masatoshi Noumi, Yasuhiko Yamada, Kanehisa Takasaki, Koji Hasegawa, Gen Kuroki and Ralph Willox for fruitful discussions and kind interest.

\section{References}

[1] M. F. de Groot, T. J. Hollowood, J. L. Miramontes, "Generalized Drinfel'd-Sokolov hierarchies", Commun. Math. Phys. 145 (1992), 57-84.

[2] V. G. Drinfel'd, V. V. Sokolov, "Lie algebras and equations of Korteweg-de Vries type", J. Sov. Math. 30 (1985), 1975-2036.

[3] V. G. Kac, D. H. Peterson, "112 Constructions of the basic representation of the loop group of $E_{8}$ " In Aymposium on anomalies, geometry and topology. W. A. Bardeen, A. R. White (eds). World Scientific, Singapore, 1985.

[4] V. G. Kac, Infinite dimensional Lie algebras, third edition. Cambridge University Press, 1990

[5] S. Leidwanger, "On the various realizations of the basic representation of $A_{n-1}^{(1)}$ and the combinatorics of partitions", J. Algebraic Combin. 14 (2001), no. 2, 133-144.

[6] M. Noumi and Y. Yamada, "Affine Weyl groups, discrete dynamical systems and Painlevé equations", Commun. Math. Phys. 199 (1998), 281-295.

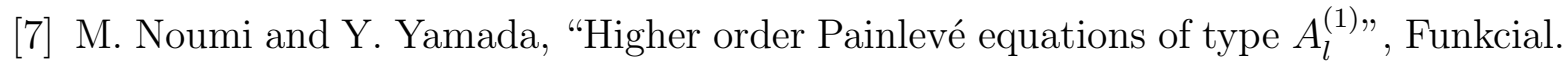
Ekvac. 41 (1998), 483-503.

[8] M. Noumi, Y. Yamada, "Symmetries in the fourth Painlevé equation and Okamoto polynomials", Nagoya Math. J. 153(1999), 53-86. 
[9] M. Noumi, "An introduction to birational Weyl group actions" in Symmetric Functions 2001: Surveys of Developments and Perspectives (Ed. S.Fomin), Proceeding of the NATO ASI held in Cambridge, U.K., June 25-July 6, 2001), 179-222, Kluwer Academic Publishers, 2002.

[10] F. ten Kroode, J. van de Leur, "Bosonic and fermionic realizations of the affine algebra $\hat{g} l_{n} "$, Commun. Math. Phys. 137 (1991), 67-107.

[11] N. Yajima, M. Oikawa, "Formation and interaction of sonic-Langmur solitons", Prog. Theor. Phys. 56 (1976), 1719-1739.

[12] Y. Yamada, "Determinant formulas for the $\tau$-functions of the Painlevé equations of type A", Nagoya Math. J. 156 (1999), 123-134. 